\title{
Dexamethasone Interferes with Autophagy and Affects Cell Survival in Irradiated Malignant Glioma Cells
}

\author{
Alfred Komakech, ${ }^{1}$ Ji-Hye Im, ${ }^{1}$ Ho-Shin Gwak, Kyue-Yim Lee, ${ }^{1}$ Jong Heon Kim, ${ }^{2}$ Byong Chul Yoo, ${ }^{2}$ Heesun Cheong, ${ }^{2}$ \\ Jong Bae Park, ${ }^{2}$ Ji Woong Kwon, ${ }^{3}$ Sang Hoon Shin, ${ }^{3}$ Heon Yoo ${ }^{3}$ \\ Department of Cancer Control,' National Cancer Center Graduate School of Cancer Science and Policy, Goyang, Korea \\ Department of Cancer Biomedical Science, ${ }^{2}$ National Cancer Center Graduate School of Cancer Science and Policy, Goyang, Korea \\ Neuro-oncology Clinic, ${ }^{3}$ National Cancer Center, Goyang, Korea
}

Objective : Radiation is known to induce autophagy in malignant glioma cells whether it is cytocidal or cytoprotective. Dexamethasone is frequently used to reduce tumor-associated brain edema, especially during radiation therapy. The purpose of the study was to determine whether and how dexamethasone affects autophagy in irradiated malignant glioma cells and to identify possible intervening molecular pathways.

Methods : We prepared p53 mutant U373 and LN229 glioma cell lines, which varied by phosphatase and tensin homolog (PTEN) mutational status and were used to make U373 stable transfected cells expressing GFP-LC3 protein. After performing cell survival assay after irradiation, the $\mathrm{IC}_{50}$ radiation dose was determined. Dexamethasone dose $(10 \mu \mathrm{M})$ was determined from the literature and added to the glioma cells 24 hours before the irradiation. The effect of adding dexamethasone was evaluated by cell survival assay or clonogenic assay and cell cycle analysis. Measurement of autophagy was visualized by western blot of LC3-I/LC3-II and quantified by the GFP-LC3 punctuated pattern under fluorescence microscopy and acridine orange staining for acidic vesicle organelles by flow cytometry.

Results : Dexamethasone increased cell survival in both U373 and LN229 cells after irradiation. It interfered with autophagy after irradiation differently depending on the PTEN mutational status : the autophagy decreased in U373 (PTEN-mutated) cells but increased in LN229 (PTEN wild-type) cells. Inhibition of protein kinase B (AKT) phosphorylation after irradiation by LY294002 reversed the dexamethasone-induced decrease of autophagy and cell death in U373 cells but provoked no effect on both autophagy and cell survival in LN229 cells. After ATG5 knockdown, radiation-induced autophagy decreased and the effect of dexamethasone also diminished in both cell lines. The diminished autophagy resulted in a partial reversal of dexamethasone protection from cell death after irradiation in U373 cells; however, no significant change was observed in surviving fraction LN229 cells.

Conclusion : Dexamethasone increased cell survival in p53 mutated malignant glioma cells and increased autophagy in PTENmutant malignant glioma cell but not in PTEN-wildtype cell. The difference of autophagy response could be mediated though the phosphatidylinositol 3-kinase/AKT/mammalian target of rapamycin signaling pathway.

Key Words : Autophagy · Dexamethasone · Glioma · PTEN · Radiation.

\footnotetext{
- Received : September 8, 2019 •Revised : November 12, 2019 •Accepted : December 18, 2019

- Address for reprints : Ho-Shin Gwak

Department of Cancer Control, National Cancer Center Graduate School of Cancer Science and Policy, 323 Ilsan-ro, Ilsandong-gu, Goyang 10408, Korea Tel : +82-31-920-1666, Fax : +82-31-920-2798, E-mail : nsghs@ncc.re.kr, ORCID : https://orcid.org/0000-0001-7175-4553
} 


\section{INTRODUCTION}

Glioblastoma multiforme (GBM), an aggressive primary brain tumor, is characterized by invasive tumor growth and remote extension within the brain at the later course of disease. Thus, surgery plays a limited role in the treatment of this tumor, and a cure remains elusive. Currently, the standard therapy for GBM is surgical removal, followed by concomitant chemoradiotherapy and adjuvant chemotherapy with temozolomide $^{10)}$.

Radiotherapy is a major cancer treatment modality for solid tumors as it kills cancer cells and shrinks the tumor either before or after surgical resection ${ }^{4)}$. However, two limitations to treatment success are the resistance of glioma cells to radiation and dose-related adverse events, resulting in poor prognosis.

Dexamethasone, a glucocorticoid steroid, has been used for decades in the treatment of malignant gliomas to reduce tumor-associated cerebral edema, especially during radiation therapy. However, the effect of dexamethasone on cancer cell growth and patient survival remains contentious. Many studies have suggested that it may protect cancer cells from cell death induced by chemotherapy/radiotherapy, thus promoting cancer cell survival $^{6,9,28,30)}$.

Autophagy is a vital catabolic mechanism where cells digest and recycle their own organelles for maintaining cellular homeostasis ${ }^{1329)}$. Glucocorticoids are known to induce catabolism as they frequently cause steroid-induced diabetes mellitus. In this context, dexamethasone is also known to induce autophagy in many cancer cells or immortalized cell lines in vitro $^{23,35,37,43)}$. Researchers have suggested that autophagy could be important in determining the response of tumor cells to anticancer therapy $^{15,24)}$. Ionizing radiation-induced autophagy in many solid tumors, including glioma, may trigger tumor cell survival or cell death ${ }^{8,27,40,41)}$. Cross-talk between apoptosis and autophagy has been suggested but remains unclear ${ }^{18,24)}$. In a previous study, we demonstrated that autophagy is partly responsible for radiation-induced cell death or apoptosis in malignant glioma cells ${ }^{17}$.

Phosphatase and tensin homolog (PTEN) is a tumor suppressor gene that is frequently mutated in GBM. It encodes a dual specificity phosphatase that negatively regulates the phosphatidylinositol 3-kinase (PI3K)/protein kinase B (AKT)/ mammalian target of rapamycin (mTOR) pathway, which is a key regulator of autophagy ${ }^{31)}$. The PI3K/AKT pathway plays an important role in cancer development, and its deregulation via the loss of PTEN function is a common occurrence in many aggressive cancers such as malignant gliomas ${ }^{7,22,31)}$. In addition, AKT promotes cell survival by inhibiting apoptosis through the phosphorylation and inactivation of several targets after exposure to chemotherapeutic agents or radiation $^{7,25,39)}$. Thus, it plays an important role in radiation-induced autophagy in glioma cells.

The purpose of this study was to determine whether dexamethasone affects autophagy and cell survival in irradiated malignant glioma cells and by what mechanism. The effect of dexamethasone on autophagy has never been evaluated in irradiated malignant glioma cells. We used two p53 mutant glioma cell lines that varied by PTEN mutational status and assessed the effect of dexamethasone on cell death and autophagy.

\section{MATERIALS AND METHODS}

The Institutional Review Board of National Cancer Center exempted any registration or permission for in vitro cell line experiment and publication of its result unless it uses animal model or patient's derived tissue. National Cancer Center exempted any informed consent for in vitro study including this study.

\section{Cell culture and irradiation}

Human glioblastoma cell lines, LN229 and U373, were obtained from the American Type Culture Collection. Cells were grown in Dulbecco's modified Eagle medium containing $10 \%$ fetal bovine serum and 1\% penicillin/streptomycin at $37^{\circ} \mathrm{C}, 5 \% \mathrm{CO}_{2}$. Cells were treated with $10 \mu \mathrm{M}$ dexamethasone sodium at the time of plating. At 24 hours after plating, they were irradiated with a Gammacell 1000 Elite Cesium ${ }^{137}$ source (MDS Nordion, Ottawa, Canada) for a calculated dose by dose rate of $0.0416 \mathrm{~Gy} / \mathrm{second}$.

\section{RNA interference}

To suppress autophagosome formation, we knocked down the autophagy-related 5 (ATG5) protein in both cell lines. Knockdown of ATG5 expression was done using small interfering RNA (SiRNA) and a negative control bought from Ambion (Life Technologies, Calsbad, CA, USA) transfected into the glioma cells using the Lipofectamine 2000 reagent (Invitrogen, Calsbad, CA, 
USA) in accordance with the manufacturer's instructions. After 24 hours, cells were used in the experiment.

\section{Cell survival assay}

Two methods of cell viability were employed : cell-counting using an automated cell counter (NanoEnTek, Seoul, Korea) after trypan blue staining and the clonogenic assay. During the survival assay, cells were plated on $60-\mathrm{mm}$ culture plates ( $2 \times 10^{4}$ cells). After 24 hours, $10 \mu \mathrm{M}$ dexamethasone was added, and 24 hours later, the cells were subjected to different doses of radiation : $0,2,5,10,15$, and $20 \mathrm{~Gy}$. Cells were then incubated at $37^{\circ} \mathrm{C}$ with regular media changes for 8-10 days until the control was $100 \%$ confluent. Cells were washed twice with phosphate-buffered saline (PBS), trypsinized with $1 \mathrm{~mL}$ trypsin-EDTA, stained with trypan blue, and counted using an automated cell counter. The percentage of viable cells was calculated from the ratio of treated cells to normal control cells. Three independent experiments were carried out, and the mean value was determined.

For the colony-forming assay, cells were plated at clonogenic density $\left(1.0 \times 10^{3}\right)$ in a $60-\mathrm{mm}$ culture plate, then treated with dexamethasone $(10 \mu \mathrm{M})$ and a radiation dose $(10 \mathrm{~Gy})$ as per the experiment setup. Cells were incubated for 8-10 days with regular media changes after treatment. Cells were fixed with $4 \%$ paraformaldehyde for 20 minutes, then stained with $0.4 \%$ crystal violet for 20 minutes, washed under running distilled water, and allowed to dry for 2 hours. A cluster of 50 cells or more was scored as a colony.

\section{Cell cycle analysis}

Cells were plated on $60-\mathrm{mm}$ plates $\left(1 \times 10^{5}\right.$ cells $)$ and pretreated with $10 \mu \mathrm{M}$ dexamethasone; 24 hours later, they were subjected to $10 \mathrm{~Gy}$ radiation during the log growth phase. At 72 hours after treatment, cells (including non-adherent cells) were collected, centrifuged twice at $1500 \mathrm{rpm}$ for 5 minutes in PBS to remove trypsin, then fixed slowly by adding drops of $70 \%$ cold ethanol and kept overnight at $4^{\circ} \mathrm{C}$.

The fixed cells were washed twice with cold PBS at 2000 rpm for 10 minutes each. Next, these cells were suspended in $500 \mu \mathrm{L}$ PI $(50 \mu \mathrm{g} / \mathrm{mL}$ in PBS) containing $100 \mu \mathrm{g} / \mathrm{mL}$ RNase A (CosmoGenetech, Seoul, Korea) and then incubated in darkness at room temperature for 30 minutes. DNA content was analyzed using a fluorescence-activated cell sorter (BD LSRFortessa; BD Biosciences, Franklin Lakes, NJ, USA) and the
FACSDiva software (BD Biosciences).

\section{Quantitative measurement of autophagy using acridine orange}

For acidic vesicular organelle (AVO) staining, pretreated cells (as described above) were incubated at $37^{\circ} \mathrm{C}$ for $30 \mathrm{~min}$ utes in a pre-warmed culture media containing acridine orange $(1 \mu \mathrm{g} / \mathrm{mL})$. After trypsinization and washing twice in PBS, pelleted cells were suspended in PBS and kept on ice, protected from light with an aluminum foil cover, and subjected to a fluorescence-activated cell sorter (BD LSRfortessa; BD Biosciences) and data analysis by BD FACSDiva software (BD Biosciences).

\section{Autophagy measurement using GFP-LC3}

Cells were transfected with a GFP-LC3 expression plasmid incorporated into the lentiviral vector using the Lipofectamine 2000 reagent (Invitrogen). Selection was made with puromycin to establish stably transfected cells expressing GFP-LC3 fluorescence, which was confirmed microscopically before the experiment. Three days following $10 \mathrm{~Gy}$ irradiation (pretreatment with $10 \mu \mathrm{M}$ dexamethasone as described above), cells were observed under a fluorescence microscope for GFP-LC3 fluorescence, and LC3-punctuated cells were counted. The average percentage of autophagic cells was calculated from the ratio of GFP-LC3-punctuated cells to normal cells only bearing GFP-LC3 fluorescence per high power field (×200).

\section{Confocal fluorescence microscope}

GFP-LC3-transfected cells $\left(1 \times 10^{5}\right)$ were plated on a glass coverslip coated with poly-D-lysine in a $60-\mathrm{mm}$ culture plate for 24 hours, then pretreated with dexamethasone. At 24 hours after pretreatment, cells were irradiated using a single $10 \mathrm{~Gy}$ dose, then incubated at $37^{\circ} \mathrm{C}$ for 3 days before analysis. Cells were fixed in $4 \%$ paraformaldehyde with DAPI staining for 30 minutes at room temperature. Fixed cells were washed with Dulbecco's PBS and mounted on a glass slide using mounting medium. The glass slide with a coverslip was inverted on a confocal microscope mounting. Photographs were taken of cells expressing GFP-LC3 protein and punctuated spots (autophagosome) were analyzed with ZEISS software (Zeiss, Gena, Germany). 


\section{Western blot analysis}

Cells were cultured on a $60-\mathrm{mm}$ plate $\left(1 \times 10^{5}\right.$ cells), treated and incubated for the required time (as described above), washed and trypsinized, and then centrifuged to form cell pellets. These pellets were lysed in a RIPA lysis buffer with proteases and phosphatase inhibitor cocktail (100X, GenDEPOT, Katy, TX, USA) for at least 30 minutes on ice, then cleared by centrifugation for 20 minutes at $12000 \mathrm{rpm}$ and $4^{\circ} \mathrm{C}$. The concentration of protein was determined using bovine serum albumin protein standard and measured with spectrophotometry at $562 \mathrm{~nm}$. The protein was heated at $95^{\circ} \mathrm{C}$ for 5 minutes and resolved by $12 \%$ and $15 \%$ polyacrylamide gel electrophoresis using mini-PROTEAN; it ran at $80 \mathrm{~V}$ for the first 30 minutes and later $125 \mathrm{~V}$ for 1 hour. The protein was transferred onto a nitrocellulose membrane at $250 \mathrm{~A}$ for 90 minutes at $4^{\circ} \mathrm{C}$ and then blocked for 1 hour with nonfat dry milk in $0.1 \%$ Tween in tris-buffered saline. The membrane was incubated at $4^{\circ} \mathrm{C}$ overnight in different primary antibodies : anti-LC3, anti-AKT, pAKT, anti-ATG5 (Novus Biologicals, Littleton, CO, USA), and anti- $\beta$-actin (Sigma-Aldrich, St. Louis, MO, USA). This membrane was then washed three times in TBST for 10 minutes and incubated with horseradish peroxidase-conjugated secondary antibody for 1 hour at room temperature. The protein complex was detected using western blot detection kit (Fisher Scientific, Waltham, MA, USA) A and B mixed in the ratio of $1: 1$ and visualized using an enhanced chemiluminescence developed on an X-ray film.

\section{Statistical analysis}

Three independent experiments were carried out (triplicate), and results were expressed as the mean \pm standard error of the mean. Statistical significance was calculated with a two-tailed unpaired Student t-test using GraphPad Prizm (ver. 6.0; GraphPad software, La Jolla, CA, USA). A p-value less than 0.05 was considered to be statistically significant.

\section{RESULTS}

\section{Dexamethasone protects glioma cells from cell death after irradiation}

We used a dexamethasone dose of $10 \mu \mathrm{M}$, which was determined by the literature as the highest clinically achievable plasma concentration ${ }^{21,33)}$, and applied a pre-determined radiation dose ranging from 2 Gy to 20 Gy. Dexamethasone alone did not affect cell proliferation at $10 \mu \mathrm{M}$ (Supplementary Fig. 1). Cell survival after irradiation was compared to evaluate the effect of pretreating with dexamethasone (Fig. 1). The addition of dexamethasone increased the cell survival significantly in both U373 ( $p=0.02)$ and LN229 ( $p=0.03$ ) cells. Based on these results, $10 \mathrm{~Gy}$ was used as the approximated inhibitory dose $50\left(\mathrm{IC}_{50}\right)$ for further experiments to observe apoptosis and autophagy as calculated $\mathrm{IC}_{50}$ values were from 8.92 to $9.52 \mathrm{~Gy}$ from the assumption of sigmoid dose-response.
U373

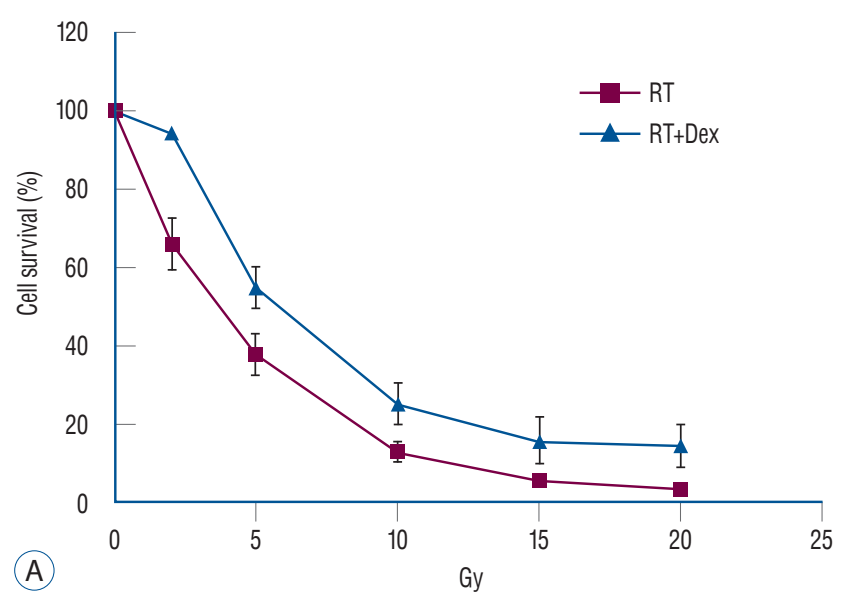

LN229

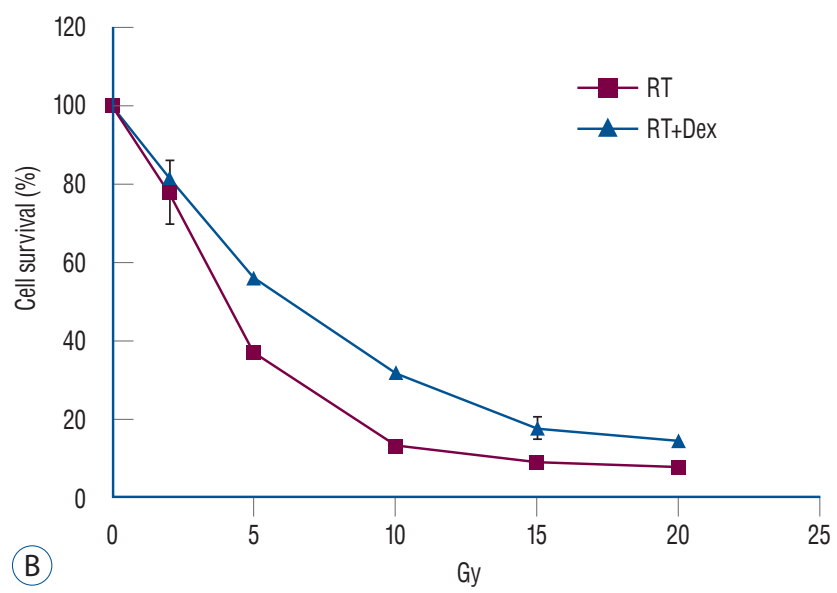

Fig. 1. Dex increases survival in both U373 and LN229 glioma cell lines after irradiation. Malignant glioma cells were pretreated with $10 \mu \mathrm{M}$ Dex and different doses of irradiation. Both U373 ( $A, p=0.02)$ and LN229 ( $B, p=0.03$ ) showed significant increases in survival. RT : radiation, Dex : dexamethasone. 

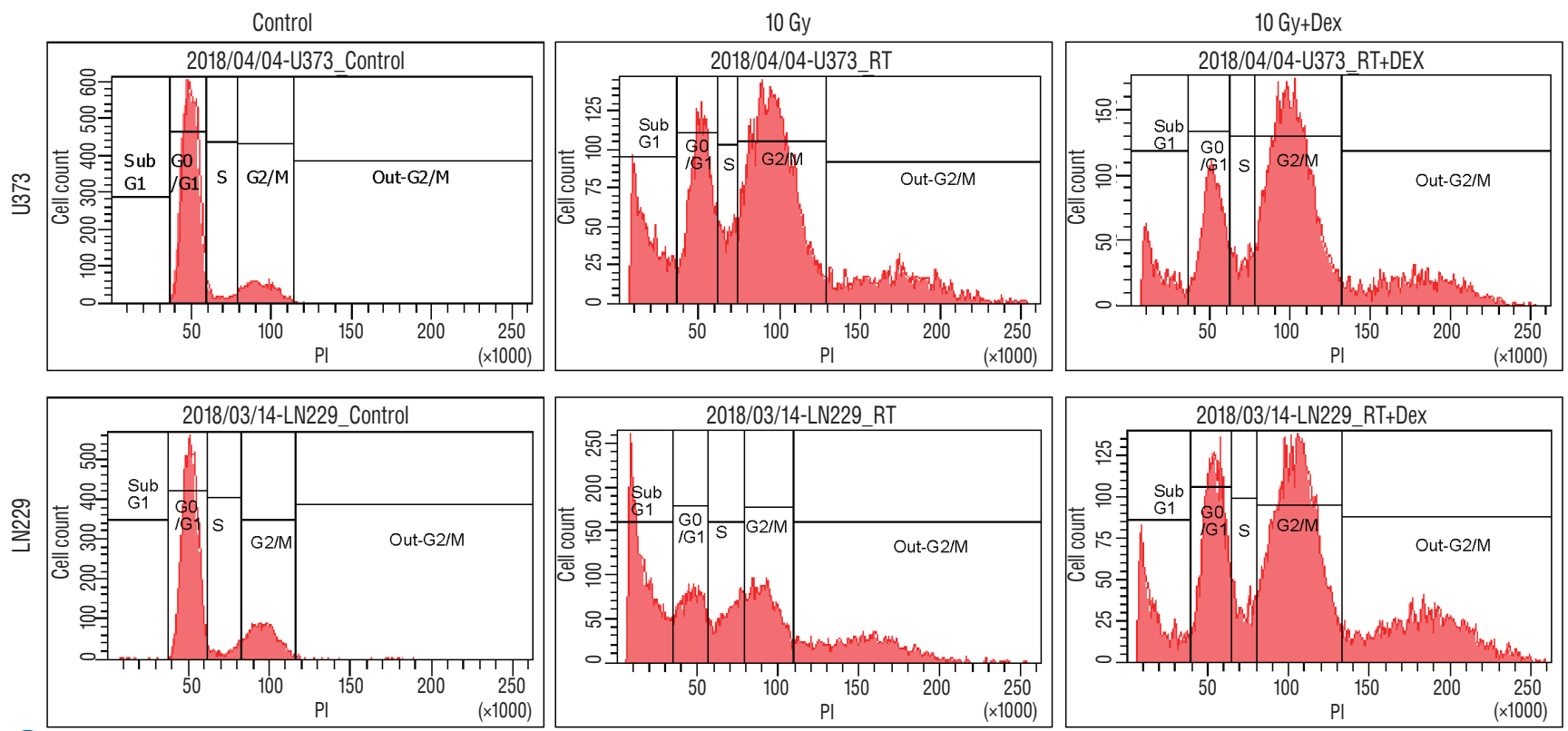

(A)

U373

LN229
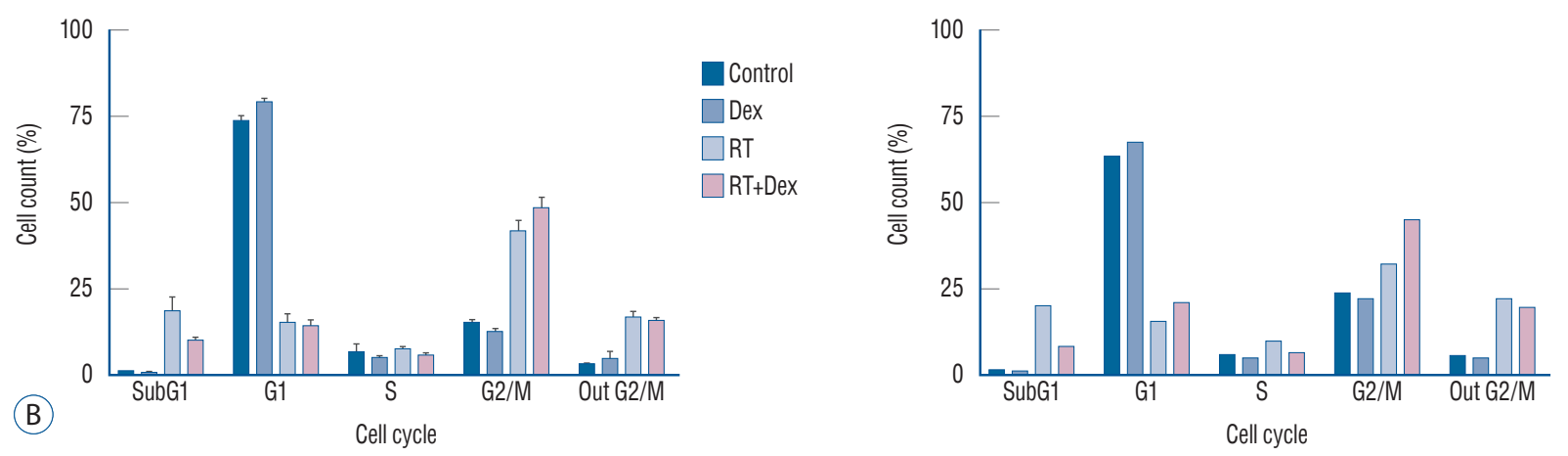

$\square$ Control
$\square$ Dex
$\square$ RT
$\square$ RT+Dex

U373

LN229
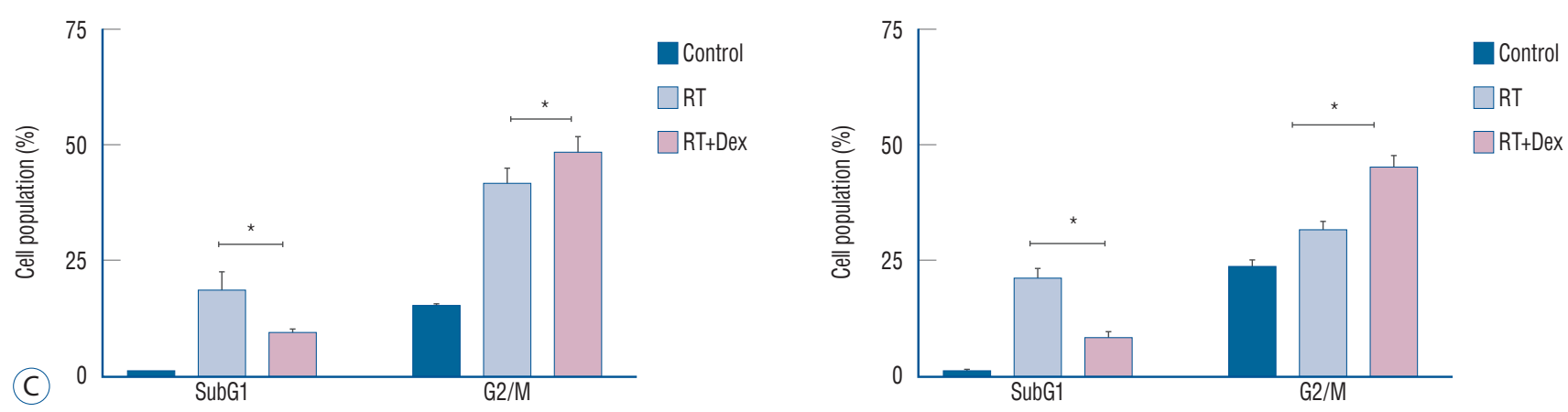

Fig. 2. Dex interferes with the cell cycle change after irradiation in both U373 and LN229 cells. A : Flow cytometry at 3 days after irradiation (10 Gy) with or without Dex. B and C : Proportional analysis of cell cycle fraction revealed radiation-induced G2/M arrest and subG1 accumulation were interrupted significantly by the addition of Dex in both U373 and LN 229. * $p<0.05$. PI : propidium iodide, RT : radiation, Dex : dexamethasone.

Cell cycle analysis after irradiation with or without dexamethasone

The steroid effect on cell cycles was observed 3 days after ir- radiation using flow cytometry analysis (Fig. 2). After $10 \mathrm{~Gy}$ irradiation, the G2/M phase accumulation was significantly increased in radiation+dexamethasone (RT+Dex) compared 


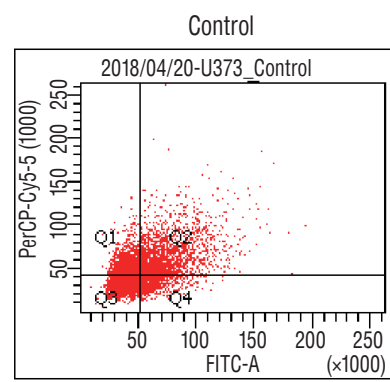

(A)

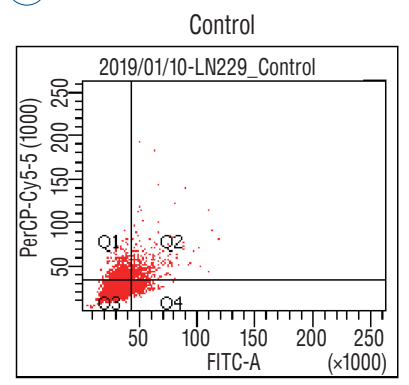

(C)

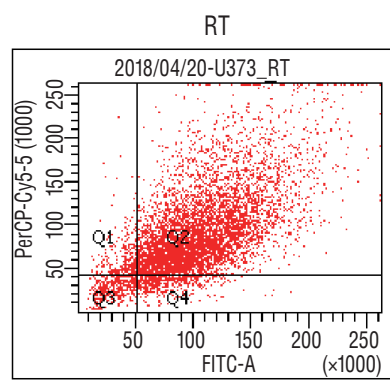

RT

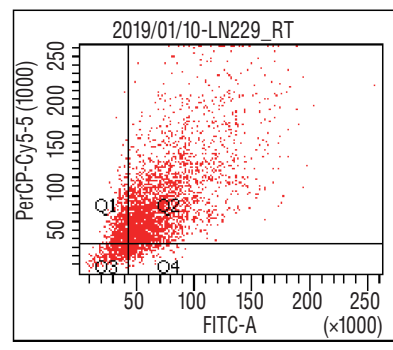

FITC-A

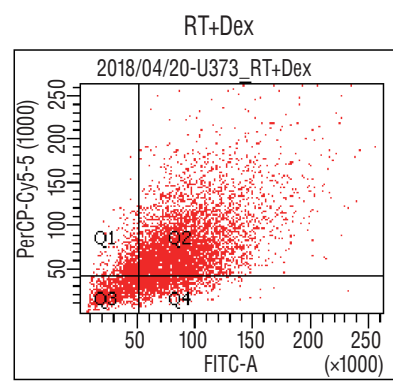

RT+Dex

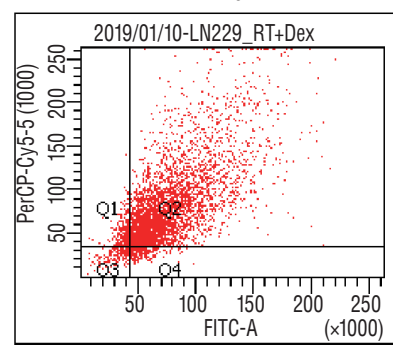

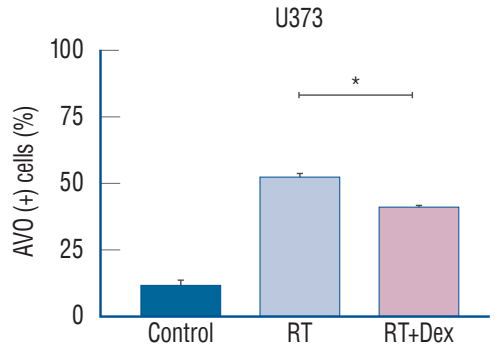

(B)

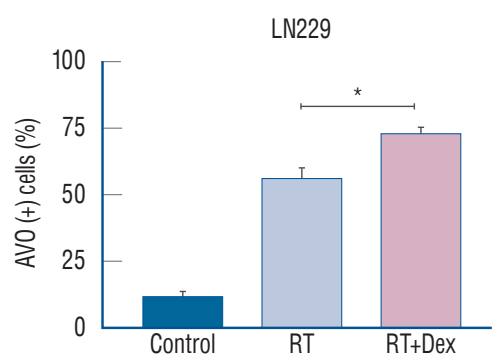

(D)

Fig. 3. Measurement of acidic vesicular organelles (AVOs) as an indicator of autophagy after irradiation (10 Gy). A and C : Quantitative analysis of AVO revealed that dexamethasone (B) decreases autophagy in U373 cells $(p=0.010)$ but (D) increases autophagy in LN229 cells $(p=0.010)$. * $p<0.05$. RT: radiation, Dex: dexamethasone, FITC-A : fluorescein isothiocyanate.

to radiation only (RT) in both U373 (48\% vs. $42 \%$ ) and LN229 ( $44 \%$ vs. $32 \%$ ) cell lines ( $p=0.021$ and 0.017 , respectively). Meanwhile, subG1 accumulation was significantly decreased with RT+Dex compared to RT in both U373 (9.8\% vs. $19 \%$, $p=0.019)$ and LN229 cells ( $8.2 \%$ vs. $20 \%, p=0.02$, respectively). These data together suggest that G2/M arrest and subG1 accumulation of a cell population at a lethal radiation dose is significantly interrupted by dexamethasone.

\section{Dexamethasone interferes autophagy after irra- diation differently according to PTEN status}

Because autophagy was a primary response to irradiation of malignant glioma cell lines in our previous study ${ }^{17}$, we measured autophagy quantitatively to evaluate the influence of dexamethasone after irradiation. After acridine orange staining of AVOs, we subjected cells to flow cytometry analysis to measure autophagy (Fig. 3). For U373 cells, the AVO-containing cells decreased significantly in RT+Dex compared with RT ( $41 \%$ vs. 52\%, $p=0.01$ ). For LN229 cells, AVO-containing cells increased as much as $56 \%$ in RT. These cells significantly increased further in $\mathrm{RT}+\mathrm{Dex}(73 \%, p=0.01)$.

The use of western blot analysis to evaluate the conversion of LC3-I to LC3-II conjugate is a standard indicator of au- tophagy $^{32)}$. In U373 cell lines, drastic reduction in the LC3-II band was found in RT+Dex compared with RT cells. By contrast, in LN229 cell lines, a significant increase of LC3-II was found in RT+Dex compared RT (Fig. 4A). These results are in accordance with quantitative measurement of AVO-indicating autophagy.

We further confirmed the inhibition of autophagy after irradiation by dexamethasone using GFP-LC3 stably transfected U373 cells (Fig. 4B). The expression level of GFP-LC3-punctuated cells showed autophagy increased after irradiation. Cells pretreated with dexamethasone (RT+Dex) exhibited less GTPLC3 granules than those treated by RT (Fig. 4C; $30 \%$ vs. 15\%, $p=0.016$ ).

Taken together, the data suggest that dexamethasone interferes with autophagy after irradiation, and the influence is different depending on the PTEN mutational status of the glioma cell.

\section{Dexamethasone-induced reduced cell death is reversed with AKT inhibitor in PTEN non-func- tional U373 cells after irradiation}

As PI3K/AKT pathway activation is the alleged cellular response to radiation-induced damage in some glioma cells ${ }^{19)}$, 


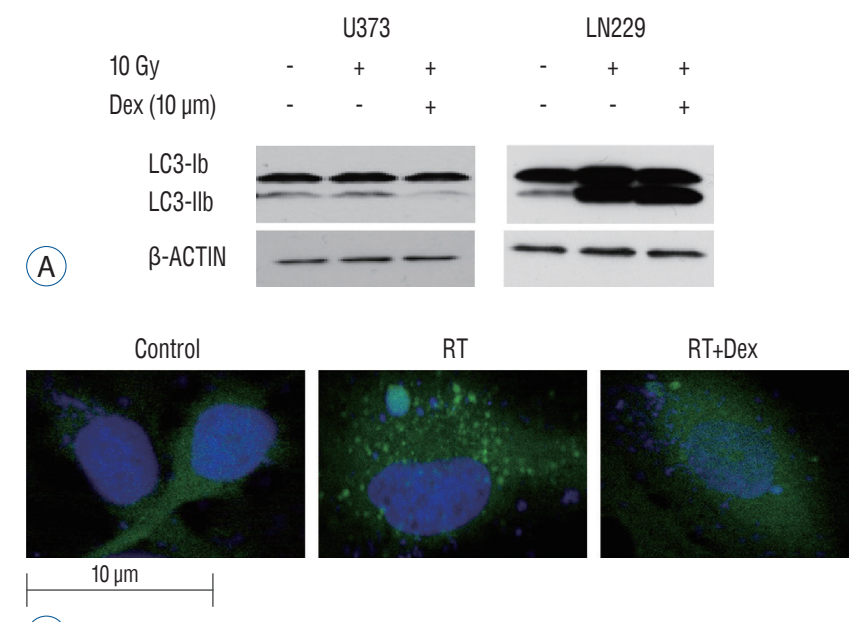

(B)

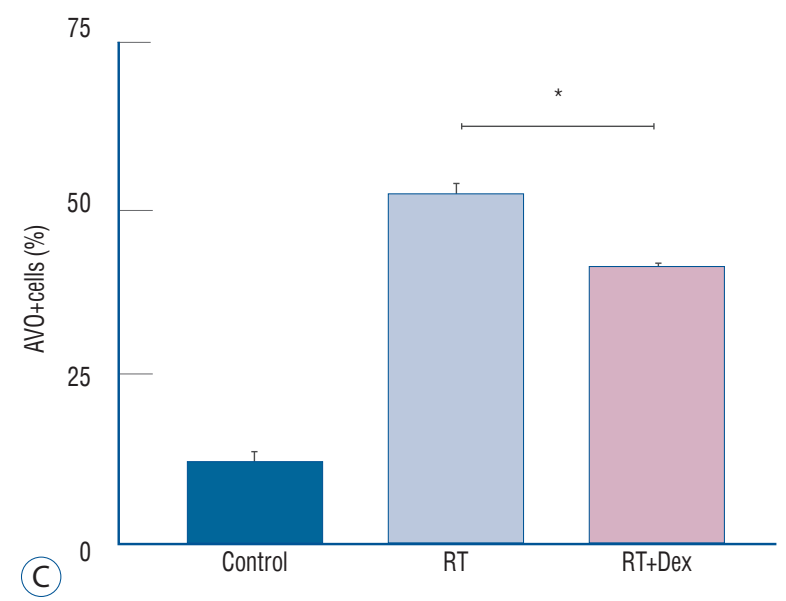

Fig. 4. Illustration of Dex's effect on radiation-induced autophagy in glioma cells. A : Dex inhibits autophagosome formation in U373 cells but promotes LC3-II conjugation in LN 229 cells on western blot. B : Fluorescence confocal microscope pictures $(\times 2000)$ showing GFP-LC3 punctuated cells after irradiation (middle), which decreased with Dex (right) in U373 cells. C : Graphs indicating reduced number of GFP-LC3 punctuated cells with Dex $(p=0.016)$ in U373 cells. Three independent experiments were carried out and the mean value is presented. ${ }^{*} p<0.05$. RT : radiation, Dex : dexamethasone, AVO : acidic vesicular organelle.

U373

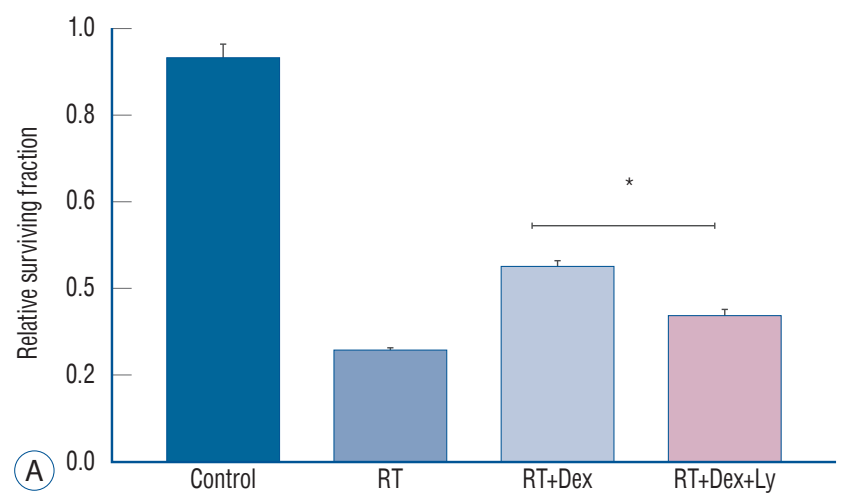

LN229

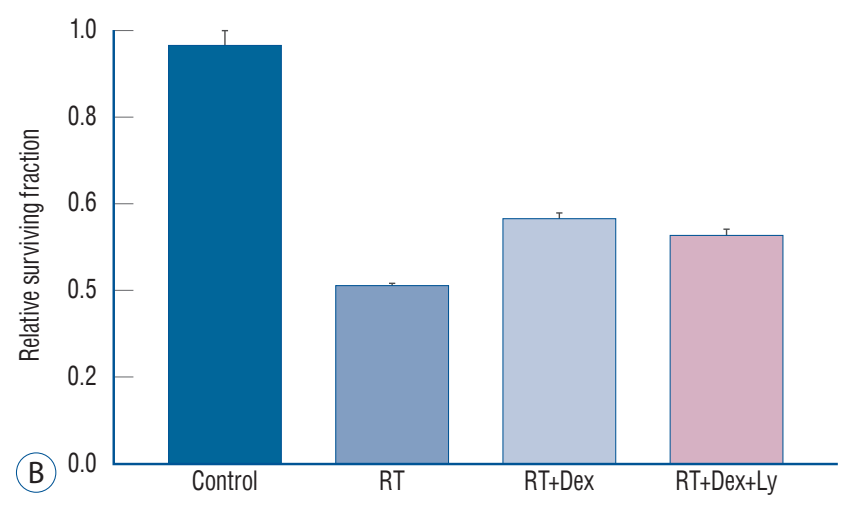

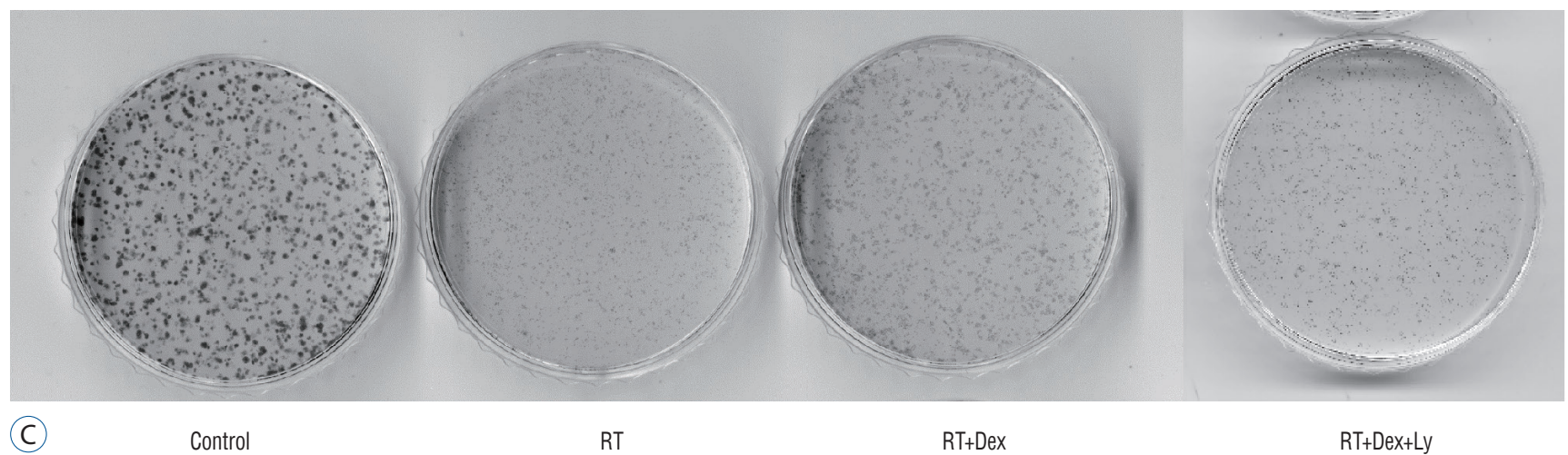

Fig. 5. Colony-forming assay after Dex and radiation exposure with or without phospho-AKT inhibitor (LY294002). A : Addition of Dex and LY294002 results in the reversal of decreased cell death with Dex in U373 cells $(p=0.0052)$. B : It provoked no significant change $(p>0.05)$ in LN229. The experiment was in triplicate and the mean value was presented. C : Photography of colony-forming assay plate of LN229 cells. ${ }^{*} p<0.05$. RT : radiation therapy, Dex : dexamethasone, LY : LY294002. 
we confirmed phosphorylation of AKT in response to radiation in PTEN-mt U373 cells but not in PTEN-wt LN229 cells (Supplementary Fig. 2). Then, we performed a colony-forming assay to determine the effect of dexamethasone on cell survival after irradiation. When dexamethasone was added to the irradiated malignant glioma cells (RT+Dex), relative cell survival fractions were significantly increased compared with RT in both U373 and LN229 cells (Fig. 5).

Next, we used the AKT inhibitor LY294002 to evaluate whether the dexamethasone effect on radiation-induced cell death is affected by the interruption of AKT activation according to PTEN status. The addition of the AKT inhibitor (RT+Dex+Ly) significantly reversed the dexamethasone-induced increased colony-forming ability in U373 cells (Fig. 5A; 0.45 vs. $0.34, p=0.0052$ ). However, the addition of LY294002 provoked no significant change in colony number in LN229 cells pretreated with dexamethasone ( $p>0.05$, Fig. $5 B)$. These data demonstrate that the dexamethasone effect on irradiated glioma cells is mediated via AKT activation in PTEN-mt glioma cells, even though autophagy activity regulated by dexamethasone treatment differs by cell line.

\section{Phospho-AKT inhibition interferes with the dexa- methasone effect on autophagy formation after irradiation in U373 glioma cells}

Next, we tested whether the effect of the AKT inhibitor on the dexamethasone-induced reduction of cell death after irradiation in U373 cells is related to autophagy activity (Fig. 6). Quantitative measurement of autophagy by AVO counting revealed that autophagy activity was significantly recovered by AKT inhibition $(\mathrm{RT}+\mathrm{Dex}+\mathrm{Ly})$ when irradiated PTEN-mt U373 cells were pretreated with dexamethasone (RT+Dex) (74\% vs. $50 \%, p=0.038$ ); no discernible change was observed in PTEN-wt LN229 cells. This increased autophagy by an
U373

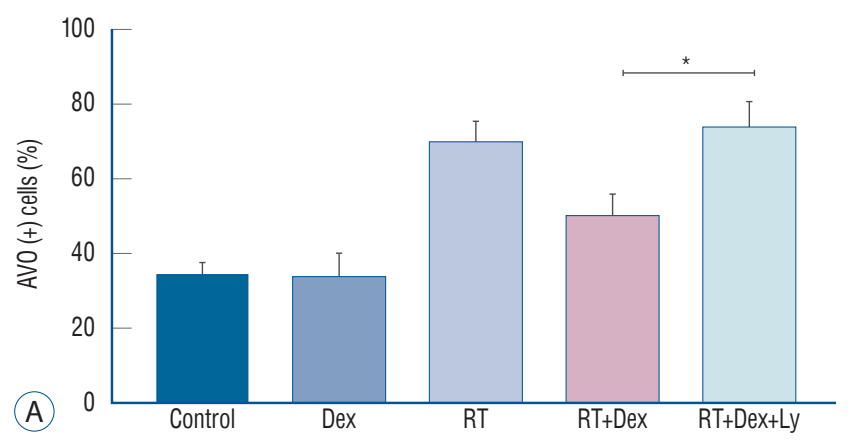

LN229

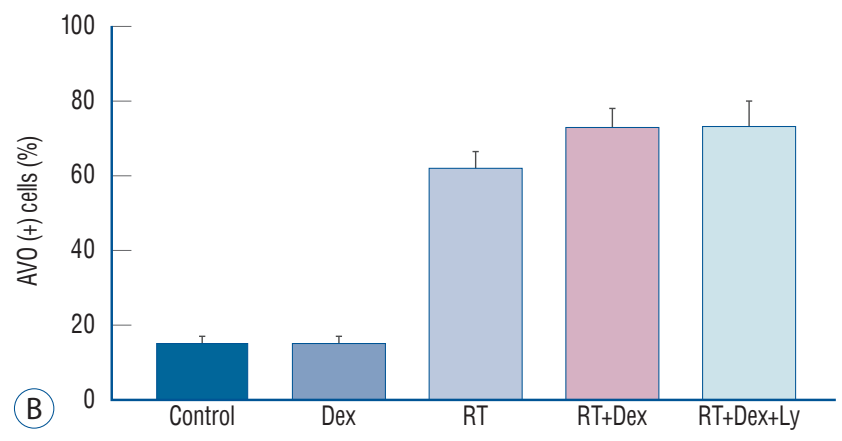

Fig. 6. Phospho-AKT inhibition affects autophagy following Dex interference in irradiated malignant glioma cells. A : In U373, AVO expression increases significantly (RT+Dex+Ly vs. RT+Dex, $p=0.038$ ), indicating increased autophagy. B : In LN229, no significant change of autophagy is seen after blockage of $\mathrm{p}-\mathrm{AKT}$. ${ }^{*} p<0.05$. AVO : acidic vesicular organelle, RT : radiation therapy, Dex : dexamethasone, LY : LY294002.

U373

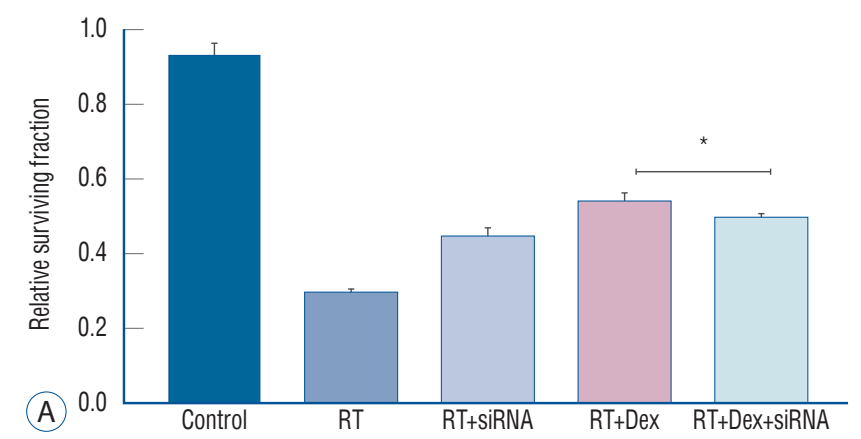

LN229

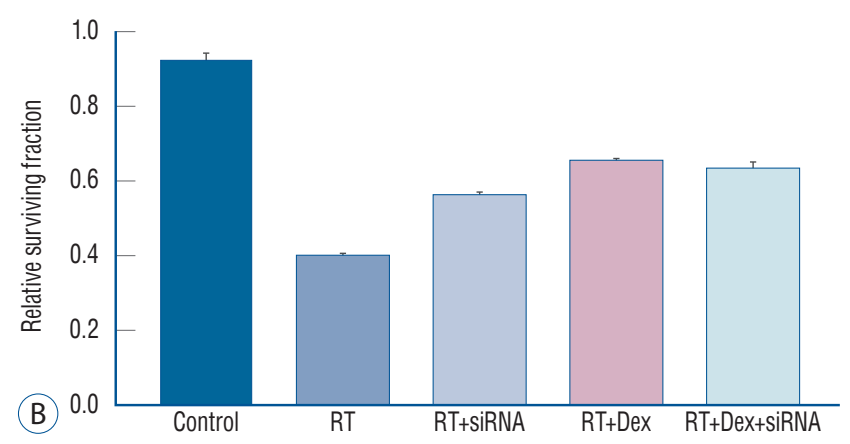

Fig. 7. After autophagy-related gene 5 (ATG5) knockdown, the Dex effect of increased colony-forming ability after irradiation is significantly reversed in PTEN-mt U373 cells but not in PTEN-wt LN229 cells. * $p<0.05$. RT : radiation therapy, Dex : dexamethasone, siRNA : small interfering RNA. 
AKT inhibitor in U373 cells appeared to reverse the autophagy inactivation induced by dexamethasone after irradiation. Altogether, these data confirm that dexamethasone may be partly acting through the PI3K/AKT/mTOR pathway in PTEN non-functional glioma cells.

\section{Autophagy inhibition diminishes dexamethasone- induced increased colony-forming ability after irradiation not in PETN-mutant U373 cells}

To determine how the knockdown of autophagy-initiating protein affect the irradiate malignant glioma cells, we knock down ATG5 protein with siRNA. After confirming ATG5 knockdown by western blot (Supplementary Fig. 3A), we measured the autophagy activity after irradiation by western blot of LC3 protein (Supplementary Fig. 3B). As expected, knock down of ATG5 significantly decreases LC3 II conjugation after irradiation in both U373 and LN229 cells.

Next, we investigated whether the inhibition of autophagy is affect reduced colony-forming ability after irradiation. We performed a colony-forming assay after transfecting cells with ATG5 siRNA (Fig. 7). The knockdown of ATG5 had no discernible effect on the clonogenic assay compared to the control (data not shown). However, ATG5 knockdown (RT+siRNA) significantly increased the colony number compared to RT ( 0.45 vs. 0.30 in U373, $p<0.001$; 0.57 vs. $0.40, p=0.005$ in LN229) in both cell lines, suggesting that autophagy plays a role on cell death after irradiation (Fig. 7). The increased colony-forming ability with dexamethasone after irradiation was diminished after ATG5 knockdown in PTEN-mt cells. In U373 cells, the colony number of the RT+Dex was significantly reduced with siRNA treatment ( 0.543 vs. $0.49, p=0.01)$. However, in LN229 cells, the colony number of the RT+Dex+siRNA was not significantly different from that of the RT+Dex (0.63 vs. $0.65, p>0.05)$. Based on these results, we assumed that the dexamethasone-induced protection from cell death after irradiation may not be mediated by autophagy in PTEN functional cells.

\section{DISCUSSION}

In our study, we found out that dexamethasone increases cell viability against radiation effect in malignant glioma cells, which was related to radio-resistance. A selective phospho-
AKT inhibitor (LY294002) resulted in the reversal of dexamethasone-induced increased cell survival after irradiation in PTEN-mt malignant glioma cells. Autophagy inhibition by ATG5 knockdown in these glioma cells resulted in the neutralization of dexamethasone-induced reduced cell death after irradiation not in PTEN-wt LN229 cells but in PTEN-mt U373 cells.

\section{Dexamethasone reduces cell death of malignant glioma cells after irradiation}

It is well-known that steroids act on glucocorticoid receptors and regulate the expression of downstream genes to exert its catabolic and anti-inflammatory effects ${ }^{26)}$. Beyond these hormonal effects, high-dose dexamethasone showed therapeutic effects against hematopoietic malignancy such as multiple myeloma and acute lymphoblastic leukemia ${ }^{2,16)}$. In addition, steroids exert a lympholytic effect on the primary central nervous system lymphoma ${ }^{38)}$. However, several studies have suggested that glucocorticoids may protect solid tumors from cytotoxic effects when used concurrently with radiotherapy or chemotherapy ${ }^{9,21)}$. Using mouse model, Pitter et al. ${ }^{28)}$ suggested that glucocorticoid use during radiation therapy was controversial and may promote cell survival in glioblastoma. In a retrospective clinical study, the administration of dexamethasone during concomitant chemoradiotherapy with temozolomide resulted in poor prognosis in newly diagnosed GBM patients $^{30)}$. Recently, Luedi et al. ${ }^{20)}$ observed the effect of dexamethasone in patient-derived GBM stem cells. They found that when exposure to dexamethasone, isocitrate dehydrogenase 1 (IDH1) wild-type cells (typical of primary GBM) showed significantly higher invasion, cell proliferation, and angiogenesis than IDH1 mutant-type GBM stem cells. In addition, they evaluated the downregulation of apoptosis-related genes and the upregulation of oncogenic genes with dexamethasone through transcriptome microarray.

In our experiment, dexamethasone reduced cell death after irradiation in both malignant glioma cell lines of the common P53 mutation, which varied by PTEN functional status. However, the molecular mechanism of dexamethasone on glioma cells either directly or after binding to the glucocorticoid receptor remains largely unknown. 


\section{Dexamethasone affects autophagy differently in irradiated malignant glioma cells according to PTEN functional status}

The uniqueness of our study was that we evaluated the effect of dexamethasone on irradiated malignant glioma cells in relation to autophagy, which has been known to be a response of tumor cells to relevant clinical doses of radiation ${ }^{12)}$. In our previous study, we suggested autophagy as a molecular response that may lead to radiation-induced cell death or apoptosis $^{17)}$.

Dexamethasone has been shown to induce autophagy in lymphocytes; however, the mechanism has yet to be fully elucidated $^{23)}$. In addition, autophagy activation was found to be secondary to dexamethasone-induced mitochondrial fragmentation in rat myoblast cells ${ }^{35}$. Wang et al. ${ }^{37)}$ suggested that dexamethasone inhibits autophagy in injured nerve cells in a dose-dependent manner.

PTEN acts as a classical tumor suppressor and is primarily involved in the homeostatic maintenance of the PI3K/AKT/ mTOR cascade, which is a key regulator of autophagy ${ }^{22)}$. PTEN is a known negative regulator of the PI3K/AKT/mTOR pathway, and PTEN expression has been found to increase autophagy in p53 mutant PTEN non-functional U87 mutant glioma cells $^{11)}$. When the effects of LY294002 on radiation were examined in the PTEN-mutant glioma cell line U251, low doses of the phospho-AKT inhibitor sensitized U251 cells to clinically relevant doses of radiation ${ }^{25}$. Andrade et al. ${ }^{3)}$ verified that dexamethasone suppresses PI3K-dependent AKT phosphorylation via glucocorticoid receptors in rat leukemic cells. Thus, we postulate that dexamethasone could be acting through glucocorticoid receptors to cause the effect observed in this study.

AKT is known to promote DNA damage repair, especially in irradiated cancer cells ${ }^{34)}$. The PI3K/AKT/mTOR pathway negatively controls autophagosome formation, particularly in PTEN non-functional cancer cells. Although we observed the same increased G2/M arrest when irradiated glioma cells were pretreated with dexamethasone, the autophagy response to radiation was different according to PTEN status. In LN229 (PTEN functional glioma) cells, the increased G2/M arrest could induce a prolonged stressful condition to cell's homeostasis that increased autophagy activity. However, in U373 (PTEN-non-functional glioma) cells, the dysregulated PI3K/ AKT/mTOR pathway may quench the stress from increased G2/M arrest into reduced autophagy as AKT phosphorylation becomes upregulated in response to radiation damage.

In this study, we observed autophagy based on acridine orange staining, western blot, and fluorescence GFP-LC3 after treating cells with dexamethasone and irradiation. Our results were in accordance with different PTEN status of tested malignant glioma cells. In U373 PTEN-mutant cells, dexamethasone inhibited autophagy after radiation; however, in PTEN wild-type LN229 cells, it increased autophagy. In addition, the phospho-AKT inhibitor LY294002 increased autophagy in PTEN-mutant U373 cells but had no effect in PTEN wildtype LN229 cells. Thus, we can postulate that dexamethasone' s effect on autophagy is mediated by phospho-AKT only in PTEN-deregulated pathways, not in PTEN functional cells.

In our study, the knockdown of ATG5 neutralized the effect of dexamethasone on autophagy after irradiation in both p53 mutant glioma cells having different PTEN status. However, the neutralization of dexamethasone's effect for protecting from radiation-induced cell death was only observed in PTEN-deregulated U373 cells. ATG5 has been characterized as a protein specifically required for autophagy; it also has a role in the formation of autophagosomes. In addition, ATG5 can be a switch molecule of cross-talk between autophagy and apoptosis as the calpain-mediated cleavage of ATG5 has proapoptotic properties ${ }^{42)}$.

The molecular mechanism of dexamethasone-induced protection from cell death after irradiation in PTEN functional LN229 remains obscure in our study. We can only suggest the radiation-induced autophagy is not directly related to cell death in this circumstance. The catabolic effect of glucocorticoids can be responsible for the increased autophagy in PTEN functional glioma cells due to another nutrient sensing pathway such as AMPK or lysosomal activation. AMPK, a cellular energy activator/sensor, is a known negative regulator of mTOR pathway ${ }^{14)}$. However, it is beyond the scope of this study. At this point and should be explored in a subsequent experiment.

\section{Possible mechanism of action of dexamethasone in irradiated glioma cells}

In our previous study, we observed that a lethal dose of radiation can induce autophagy and that G2/M fraction accumulates in proportion to the radiation dose ${ }^{17)}$. Thus, we can suggest that DNA damage provoking G2/M arrest after irradiation was strong enough to increase autophagy in glioma cells. 
Tusher et al. ${ }^{36)}$ identified a major change of gene expression after radiation using microarray in lymphoma cell lines. Among significantly upregulated genes after irradiation, 60\% were genes related to cell cycle regulation, and 9\% were involved in apoptosis. G2/M arrest activates corresponding cyclin-dependent kinases, which in turn activate repair machinery of DNA double-strand break, a common radiation injury". In our study, increased G2/M arrest and decreased subG1 fraction with dexamethasone pretreatment may be a result of increased DNA double-strand break repair. Aasland et al. ${ }^{1)}$ had demonstrated that $\mathrm{O}^{6}$-methylguanine-DNA methyltransferase (MGMT), which is a significant biomarker for glioblastoma concomitant chemoradiotherapy with temozolomide, was induced by glucocorticoids because the MGMT promotor site has two glucocorticoid response elements (GRE) in HeLa S3 cells. Later, they evaluated that MGMT expression is not affected by radiation or temozolomide and is primarily controlled by specificity protein 1 (SP1) transcription factor in various malignant glioma cell lines. In addition, they suggested that p53 activation after irradiation suppresses its downstream target SP1, elevating the important role of glucocorticoid on MGMT expression via GRE binding in p53 mutant glioma cells.

Unfortunately, we neither quantified DNA damage after irradiation nor evaluated the expression of corresponding cyclin-dependent kinases. Thus, we could not say whether the dexamethasone protection from radiation-induced cell death in our study was from decreased DNA damage or increased DNA damage repair. We need to evaluate this point in future experiments, which will be dedicated to dexamethasone-induced DNA repair machinery change and responsible molecular pathway.

\section{CONCLUSION}

In our study, PTEN mutational status of malignant glioma cells is responsible for the different autophagy interference of dexamethasone after radiation. Autophagy inhibition after ATG5 knockdown reduces cell death or apoptosis only in PTEN non-functional glioma cells. Dexamethasone interference of autophagy observed in malignant glioma cells during our experiment seems to be partly mediated through the $\mathrm{PI} 3 \mathrm{~K} / \mathrm{AKT} / \mathrm{mTOR}$ pathway in PTEN-mt cells.
Our findings demonstrated that the combined treatment of dexamethasone with radiation in malignant glioma cells reduces cell death, which could be responsible for the radio-resistance frequently observed during radiotherapy in patients with malignant gliomas. Careful use of dexamethasone during radiotherapy could be important to minimize treatment failure in glioblastoma patients.

\section{CONFLICTS OF INTEREST}

No potential conflict of interest relevant to this article was reported.

\section{INFORMED CONSENT}

This type of study does not require informed consent.

\section{AUTHOR CONTRIBUTIONS}

Conceptualization : JHI, HSG, KYL, JHK, BCY, HSC, JBP, JWK, SHS, HY

Data curation : AK, HSG, KYL

Formal analysis : AK, JHI, HSG, KYL, HSC

Funding acquisition : HSG, JHK, BCY, JBP, JWK

Methodology : AK, JHI, HSG, KYL, JHK, BCY, HSC, JBP, JWK

Project administration : HSG, JBP, JWK, SHS, HY

Visualization : AK, JHI, HSG, KYL, HSC, JBP

Writing - original draft : AK, JHI, HSG, KYL, HSC

Writing - review \& editing: AK, JHI, HSG, KYL, JHK, BCY, HSC, JBP, JWK, SHS, HY

\section{ORCID}

Alfred Komakech https://orcid.org/0000-0002-8423-911X Ji-Hye Im https://orcid.org/0000-0002-3941-4134

Ho-Shin Gwak https://orcid.org/0000-0001-7175-4553

Kyue-Yim Lee https://orcid.org/0000-0003-4050-1523

Jong Heon Kim https://orcid.org/0000-0002-1091-8099 
Byong Chul Yoo

Heesun Cheong

Jong Bae Park

Ji Woong Kwon

Sang Hoon Shin

Heon Yoo

https://orcid.org/0000-0001-9726-9492

https://orcid.org/0000-0003-1946-107X

https://orcid.org/0000-0003-0207-0697

https://orcid.org/0000-0002-5777-5398

https://orcid.org/0000-0002-8404-1162

https://orcid.org/0000-0002-9223-4300

\section{- Acknowledgements}

A part of this study was presented at the 13th Winter Meeting of Korean Brain Tumor Society (2019 January 25).

This work was supported by grants from National Cancer Center, Korea (NCC1710871-3 and 1910090-2), and a grant of the Korea Health Industry Development Institute, funded by the Ministry of Health \& welfare, Republic of Korea (HI17C1018).

The first author (A.K.) sincerely thanks the Korea Foundation for International Healthcare (KOFIH) and the Uganda Cancer Institute (UCI) for giving him the opportunity to perform this study and supporting him throughout his training program.

\section{- Supplementary materials}

The online-only data supplement is available with this article at https://doi.org/10.3340/jkns.2019.0187.

\section{References}

1. Aasland D, Reich TR, Tomicic MT, Switzeny OJ, Kaina B, Christmann M : Repair gene $0^{6}$-methylguanine-DNA methyltransferase is controlled by SP1 and up-regulated by glucocorticoids, but not by temozolomide and radiation. J Neurochem 144 : 139-151, 2018

2. Alexanian R, Dimopoulos MA, Delasalle K, Barlogie B : Primary dexamethasone treatment of multiple myeloma. Blood 80 : 887-890, 1992

3. Andrade MV, Hiragun T, Beaven MA : Dexamethsaone suppresses antigen-induced activation of phosphatidylinositol 3-kinase and downstream responses in mast cells. J Immunol 172 : 7254-7262, 2004

4. Baskar R, Dai J, Wenlong N, Yeo R, Yeoh KW : Biological response of cancer cells to radiation treatment. Front Mol Biosci 1 : 24, 2014

5. Branzei D, Foiani $M$ : Regulation of DNA repair throughout the cell cycle. Nat Rev Mol Cell Biol 9 : 297-308, 2008

6. Cenciarini M, Valentino M, Belia S, Sforna L, Rosa P, Ronchetti S, et al. : Dexamethasone in glioblastoma multiforme therapy: mechanisms and controversies. Front Mol Neurosci $12: 65,2019$

7. Chen M, Nowak DG, Trotman LC : Molecular pathways: PI3K pathway

phosphatases as biomarkers for cancer prognosis and therapy. Clin Cancer Res 20 : 3057-3063, 2014

8. Classen F, Kranz P, Riffkin H, Pompsch M, Wolf A, Göpelt K, et al. : Autophagy induced by ionizing radiation promotes cell death over survival in human colorectal cancer cells. Exp Cell Res 374 : 29-37, 2019

9. Das A, Banik NL, Patel SJ, Ray SK : Dexamethasone protected human glioblastoma U87MG cells from temozolomide induced apoptosis by maintaining Bax:Bcl-2 ratio and preventing proteolytic activities. Mol Cancer $3: 36,2004$

10. Davis ME : Glioblastoma: overview of disease and treatment. Clin J Oncol Nurs 20(5 Suppl) : S2-S8, 2016

11. Errafiy R, Aguado C, Ghislat G, Esteve JM, Gil A, Loutfi M, et al. : PTEN increases autophagy and inhibits the ubiquitin-proteasome pathway in glioma cells independently of its lipid phosphatase activity. PLoS One 8 : e83318, 2013

12. Gewirtz DA : The autophagic response to radiation: relevance for radiation sensitization in cancer therapy. Radiat Res 182 : 363-367, 2014

13. Gustafsson $A B$, Gottlieb RA : Recycle or die: the role of autophagy in cardioprotection. J Mol Cell Cardiol 44 : 654-661, 2008

14. Herzig S, Shaw RJ : AMPK: guardian of metabolism and mitochondrial homeostasis. Nat Rev Mol Cell Biol 19 : 121-135, 2018

15. Hippert MM, O'Toole PS, Thorburn A : Autophagy in cancer: good, bad, or both? Cancer Res 66 : 9349-9351, 2006

16. Inaba $\mathrm{H}$, Pui $\mathrm{CH}$ : Glucocorticoid use in acute lymphoblastic leukaemia. Lancet Oncol 11 : 1096-1106, 2010

17. Jo GH, Bögler O, Chwae YJ, Yoo H, Lee SH, Park JB, et al. : Radiationinduced autophagy contributes to cell death and induces apoptosis partly in malignant glioma cells. Cancer Res Treat 47 : 221-241, 2015

18. Kim KW, Mutter RW, Cao C, Albert JM, Freeman M, Hallahan DE, et al. : Autophagy for cancer therapy through inhibition of pro-apoptotic proteins and mammalian target of rapamycin signaling. J Biol Chem 281 : 36883-36890, 2006

19. Li HF, Kim JS, Waldman T : Radiation-induced Akt activation modulates radioresistance in human glioblastoma cells. Radiat Oncol $4: 43$, 2009

20. Luedi MM, Singh SK, Mosley JC, Hassan ISA, Hatami M, Gumin J, et al. : Dexamethasone-mediated oncogenicity in vitro and in an animal model of glioblastoma. J Neurosurg 129 : 1446-1455, 2018

21. Mattern J, Büchler MW, Herr I : Cell cycle arrest by glucocorticoids may protect normal tissue and solid tumors from cancer therapy. Cancer Biol Ther 6 : 1345-1354, 2007

22. Milella M, Falcone I, Conciatori F, Cesta Incani U, Del Curatolo A, Inzerilli $\mathrm{N}$, et al. : PTEN: multiple functions in human malignant tumors. Front Oncol 5 : 24, 2015

23. Molitoris JK, McColl KS, Swerdlow S, Matsuyama M, Lam M, Finkel TH, et al. : Glucocorticoid elevation of dexamethasone-induced gene 2 (Dig2/ RTP801/REDD1) protein mediates autophagy in lymphocytes. J Biol Chem 286 : 30181-30189, 2011

24. Moretti L, Attia A, Kim KW, Lu B : Crosstalk between Bak/Bax and mTOR signaling regulates radiation-induced autophagy. Autophagy 3 : 142-144, 2007 
25. Nakamura JL, Karlsson A, Arvold ND, Gottschalk AR, Pieper RO, Stokoe $D$, et al. : PKB/Akt mediates radiosensitization by the signaling inhibitor LY294002 in human malignant gliomas. J Neurooncol 71 : 215-222, 2005

26. Oakley RH, Cidlowski JA : The biology of the glucocorticoid receptor: new signaling mechanisms in health and disease. J Allergy Clin Immunol 132 : 1033-1044, 2013

27. Paglin S, Hollister T, Delohery T, Hackett N, McMahill M, Sphicas E, et al. : A novel response of cancer cells to radiation involves autophagy and formation of acidic vesicles. Cancer Res 61 : 439-444, 2001

28. Pitter KL, Tamagno I, Alikhanyan K, Hosni-Ahmed A, Pattwell SS, Donnola $S$, et al. : Corticosteroids compromise survival in glioblastoma. Brain 139 : 1458-1471, 2016

29. Ravikumar B, Sarkar S, Davies JE, Futter M, Garcia-Arencibia M, GreenThompson ZW, et al. : Regulation of mammalian autophagy in physiology and pathophysiology. Physiol Rev 90 : 1383-1435, 2010

30. Shields LB, Shelton BJ, Shearer AJ, Chen L, Sun DA, Parsons S, et al. : Dexamethasone administration during definitive radiation and temozolomide renders a poor prognosis in a retrospective analysis of newly diagnosed glioblastoma patients. Radiat Oncol 10 : 222, 2015

31. Song MS, Salmena L, Pandolfi PP : The functions and regulation of the PTEN tumour suppressor. Nat Rev Mol Cell Biol 13 : 283-296, 2012

32. Tanida I, Ueno T, Kominami E : LC3 and autophagy. Methods Mol Biol $445: 77-88,2008$

33. Tóth GG, C Kloosterman C, Uges DR, Jonkman MF : Pharmacokinetics of high-dose oral and intravenous dexamethasone. Ther Drug Monit 21 : 532-535, 1999

34. Toulany $M$, Lee KJ, Fattah KR, Lin YF, Fehrenbacher $B$, Schaller $M$, et al. : Akt promotes post-irradiation survival of human tumor cells through initiation, porgression, and termination of DNA-PKcs-dependent DNA double-strand break repair. Mol Cancer Res 10 : 945-957, 2012

35. Troncoso R, Paredes F, Parra V, Gatica D, Vasquez-Trincado C, Quiroga $C$, et al. : Dexamethasone-induced autophagy mediates muscle atrophy through mitochondrial clearance. Cell Cycle 13 : 2281-2295, 2014

36. Tusher VG, Tibshirani R, Chu G : Significance analysis of microarrays applied to the ionizing radiation response. Proc Natl Acad Sci U S A 98 : 5116-5121, 2001

37. Wang Z, Zhou L, Zheng X, Liu W : Effects of dexamethasone on autophagy and apoptosis in acute spinal cord injury. Neuroreport 29 : 10841091, 2018

38. Weller $\mathrm{M}:$ Glucocorticoid treatment of primary CNS lymphoma. J Neurooncol 43 : 237-239, 1999

39. Willers H, Azzoli CG, Santivasi WL, Xia F : Basic mechanisms of therapeutic resistance to radiation and chemotherapy in lung cancer. Cancer J 19 : 200-207, 2013

40. Wilson EN, Bristol ML, Di X, Maltese WA, Koterba K, Beckman MJ, et al. : A switch between cytoprotective and cytotoxic autophagy in the radiosensitization of breast tumor cells by chloroquine and vitamin D. Horm Cancer 2 : 272-285, 2011

41. Yao KC, Komata T, Kondo Y, Kanzawa T, Kondo S, Germano IM : Molecular response of human glioblastoma multiforme cells to ionizing radiation: cell cycle arrest, modulation of the expression of cyclin-dependent kinase inhibitors, and autophagy. J Neurosurg 98 : 378-384, 2003

42. Yousefi S, Perozzo R, Schmid I, Ziemiecki A, Schaffner T, Scapozza L, et al. : Calpain-mediated cleavage of Atg5 switches autophagy to apoptosis. Nat Cell Biol 8 : 1124-1132, 2006

43. Zhang S, Liu Y, Liang Q : Low-dose dexamethasone affects osteoblast viability by inducing autophagy via intracellular ROS. Mol Med Rep 17 : 4307-4316, 2018 